Article

\title{
Genome-Wide Identification and Characterization of Lectin Receptor-Like Kinase Gene Family in Cucumber and Expression Profiling Analysis under Different Treatments
}

\author{
Duo Lv, Gang Wang, Liang-Rong Xiong, Jing-Xian Sun, Yue Chen, Chun-Li Guo, Yao Yu, \\ Huan-Le He, Run Cai and Jun-Song Pan * \\ School of Agriculture and Biology, Shanghai Jiao Tong University, Shanghai 200240, China; \\ lvduocloudy@sjtu.edu.cn (D.L.); wg@shu.edu.cn (G.W.); xlr1394981178@sjtu.edu.cn (L.-R.X.); \\ leyunu@sjtu.edu.cn (J.-X.S.); yuechen321@sjtu.edu.cn (Y.C.); jackling@sjtu.edu.cn (C.-L.G.); \\ yuyaosjtu@sjtu.edu.cn (Y.Y.); hlhe75@sjtu.edu.cn (H.-L.H.); cairun@sjtu.edu.cn (R.C.) \\ * Correspondence: jspan71@sjtu.edu.cn
}

Received: 22 July 2020; Accepted: 31 August 2020; Published: 2 September 2020

\begin{abstract}
Lectin receptor-like kinases (LecRLKs) are a class of membrane proteins found in plants that are involved in diverse functions, including plant development and stress responses. Although LecRLK families have been identified in a variety of plants, a comprehensive analysis has not yet been undertaken in cucumber (Cucumis sativus L.). In this study, 46 putative LecRLK genes were identified in the cucumber genome, including 23 G-type and 22 L-type, and one C-type LecRLK gene. They were unequally distributed on all seven chromosomes, with a clustering tendency. Most of the genes in the cucumber LecRLK (CsLecRLK) gene family lacked introns. In addition, there were many regulatory elements associated with phytohormones and stress on these genes' promoters. Transcriptome data demonstrated distinct expression patterns of CsLecRLK genes in various tissues. Furthermore, we found that each member of the CsLecRLK family had its own unique expression pattern under hormone and stress treatment by the quantitative real-time PCR (qRT-PCR) analysis. This study provides a better understanding of the character and function of the LecRLK gene family in cucumber and opens up the possibility to exploring the roles that LecRLKs might play in the life cycle of cucumber.
\end{abstract}

Keywords: cucumber; expression analysis; genome-wide analysis; lectin receptor-like kinase; phylogenetic analysis

\section{Introduction}

In order to better adapt to the living environment, plants have evolved a complete set of signal receptor proteins over the course of their evolution. After receiving external stimulus, they transmit signals to downstream pathways to allow plants to respond to the stimulus. Cell-surface receptors, a kind of signal receptor proteins, play important roles in receiving and transmitting environmental signals. The receptor-like kinase (RLK) family, one important family of cell surface receptors, contains three domains, such as the extracellular domain, transmembrane domain (TM) and intracellular kinase domain [1]. RLK proteins could be classified into different families based on the structure of the extracellular domains [2].

Lectin receptor-like kinases (LecRLKs), a class of RLKs that contain a lectin domain within the extracellular domain [1], are a gene family that is specialized for sensing external environmental stimuli and transmitting signals. They are localized on the cell membrane, relying on $\mathrm{N}$ terminus diverse 
extracellular ligand recognition domains (also called lectin domain) to recognize various environmental stimuli, and then phosphorylate downstream proteins through their $\mathrm{C}$ terminus intracellular kinase domain to pass on received signals [1,3].

Based on the identity of the lectin domains, the LecRLKs have been divided into three subfamilies (Figure 1): L-type, G-type and C-type LecRLKs [4]. These subclasses are very distinct from each other, differentiated by the sugar-binding ability of the lectin domain. The G-type LecRLKs were previously named the B-type LecRLKs, due to the resemblance of their extracellular domains to bulb lectin proteins [5]. G-type LecRLKs were also historically known as S-domain RLKs, because apart from containing bulb lectin protein, its extracellular domain sometimes contains an S-locus region, known for its role in self-incompatibility reactions [5,6]. In most proteins (but not necessarily in all), they are also accompanied by an Epidermal Growth Factor (EGF) motif and/or a Plasminogen Apple Nematode (PAN) motif [4] (Figure 1B). The EGF motif contains some cysteines and is involved in the formation of disulfide bonds [4,7]. The PAN motif is believed to be related to protein-protein and protein-carbohydrate interactions [8]. As the name suggests, the lectin domain of L-type LecRLKs resembles the soluble lectin protein found in leguminous plants [9]. The third class of LecRLKs is C-type lectin kinases. The C-type LecRLKs in plants are thought to be homologues of calcium-dependent lectin motifs, which are a large group of mammalian proteins known to be involved in innate immune responses and pathogen recognition [10]. Although C-type lectins are present in large numbers in mammalian systems, C-type LecRLKs seem to be scarce in plants [3]. For example, only one gene encoding for C-type LecRLK exists in both rice and Arabidopsis [4].

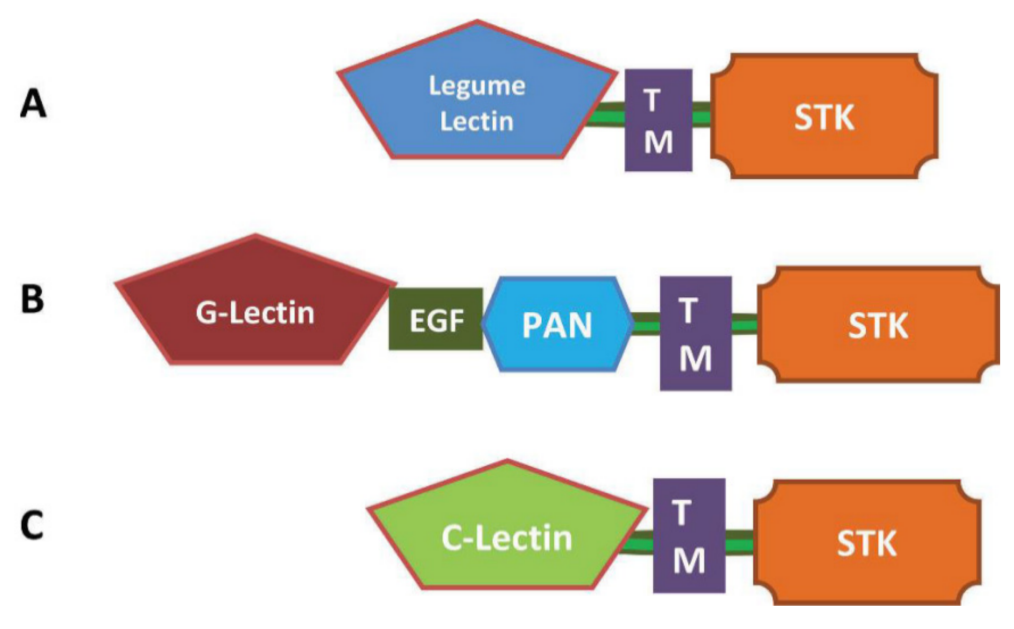

Figure 1. Line Model of the three Lectin receptor-like kinases (LecRLKs) [4]. (A) L-type LecRLKs; (B) G-type LecRLKs; (C) C-type LecRLKs. TM: transmembrane region; STK: cytoplasmic Serine/Threonine kinase domain. PAN: Plasminogen Apple Nematode domain; EGF: Epidermal Growth Factor domain.

At present, the existing research on the biological functions of LecRLKs is not very extensive [5]. Some studies suggest that LecRLKs are mainly involved in plant development, stress response and innate immune responses. For example, LecRK-b2, an L-type receptor-like kinase in Arabidopsis, can be induced by abscisic acid, salt and osmotic stress [11]. The promoter of LecRK- $b 2$ can be activated by transcription factor ABSCISIC ACID INSENSITIVE3, which mediates abscisic acid (ABA) responses in seeds [11]. Another L-type receptor-like kinase, LECRK-IV.2, plays an important role in the sterility of Arabidopsis. In the mutant of LECRK-IV.2, all pollen grains are deformed and collapsed [12]. In rice, OslecRK can maintain seed viability by regulating the expression of $\alpha$-amylase genes. The mutant of OslecRK reduces the resistance of rice plants to fungal and bacterial pathogens as well as herbivorous insects [13]. 
Genome-wide analysis of the LecRLK gene family has been reported for some plants [4,14-16], but there are only a few early studies reporting on the presence of LecRLKs in Cucurbitaceae crops [17]. A comprehensive understanding of LecRLK genes in cucumber is still lacking. In this study, we used bioinformatics methods to identify LecRLK genes from the cucumber genome and analyzed their phylogenetic relationships, gene structure, conserved domain, gene duplications, chromosome distribution and cis-acting elements on the promoters. Finally, we profiled the expression of the predicted genes in different tissues and response to gibberellin (GA), abscisic acid (ABA), 1-Naphthaleneacetic acid (NAA), indole-3-acetic acid (IAA) and cold treatments in cucumber. Our study provides valuable information for further functional research on the LecRLK gene family in cucumber.

\section{Materials and Methods}

\subsection{Identification of LecRLK Genes in Cucumber}

The whole genome and protein sequence data of cucumber were downloaded from a public database (http://cucurbitgenomics.org/; Cucumber (Chinese Long) genome v2). The Hidden Markov Model (HMM) was used to identify cucumber LecRLK candidates, and the HMM profiles of LecRLKs were downloaded from the Pfam protein database (http://pfam.xfam.org/). The models for these files were L-type (Lectin_legB PF00139), G-type (B_lectin PF01453) and C-type (Lectin_C PF00059). We used HMMER 3.0 [18] to search three types of genes from cucumber protein sequence data with e-value cutoffs of $<0.001$. We then submitted protein sequences of these genes to two bioinformatics websites, Pfam [19] (http://pfam.xfam.org/) and SMART (2019) [20] (http://smart.embl.de/), with an e-value cut-off of 1.0, while retaining those genes that contained lectin domain, transmembrane domains and kinase domains at the same time. The LecRLK family data of rice and Arabidopsis were taken from a previous research literature [4].

\subsection{Phylogenetic Analysis}

The full-length protein sequences of CsLecRLKs were aligned using the MUSCLE program (http://www.ebi.ac.uk/Tools/msa/muscle/) with the default parameters [21]. The phylogenetic tree was constructed through the Neighbor-Joining (NJ) method using MEGAX_software v.10.1.8 with the following parameters: Poisson model, pairwise deletion and 1000 bootstrap replications. The phylogram was rooted using two distantly related receptor kinase (RK) sequences of cucumber, CsaLRR-RK (Csa3G126190) and CsaPERK1 (Csa2G004170). We also chose At1G52310 (C-type LecRLK of Arabidopsis), At4G04960 (L-type LecRLK of Arabidopsis) and At1G61550 (G-type LecRLK of Arabidopsis) as outgroups of each LecRLK type.

\subsection{Conserved Domain, Motif Identification and Gene Structure Analysis}

The conserved motifs of the CsLecRLKs were predicted using the MEME program v.5.1.1 [22] (http://meme-suite.org/). The parameters were set as any number of repetitions, an optimum motif width of 6-210 residues, and 10 motifs were searched for. The site distribution mode was "zoops", and the maximum search time was 18,000 s. The CDD program (https://www.ncbi.nlm.nih.gov/cdd/) was used to predict the function of each conservative motif with an e-value threshold of 0.01 . Gene Structure Display Server [23] (http://gsds.cbi.pku.edu.cn/) was used to show the exon-intron structures of CsLecRLK genes. Adobe Illustrator CS6 was used to enhance figures.

\subsection{Gene Location and Duplication Analysis of CsLecRLKs}

A series of in-house Perl scripts was used to retrieve the location information for each CsLecRLK and the length of each cucumber chromosome from cucumber whole-genome sequence data, which were downloaded from a public database (http://cucurbitgenomics.org/) (Cucumber (Chinese Long) genome v2). Location information of each CsLecRLK and length of each chromosome were then 
displayed as visualized pictures through MapChart [24]. The Adobe Illustrator CS6 was used to enhance the picture.

We used two methods to find duplication events among the CsLecRLKs. One was confirming gene duplication with three criteria using in-house Perl scripts: (a) the shorter aligned gene covered $>70 \%$ of the longer gene in length, (b) the similarity of aligned sequences was $>70 \%[25,26]$ and (c) two genes located in the same chromosomal fragment of less than $100 \mathrm{~kb}$ and separated by five or fewer genes were identified as tandem duplicated genes [27]. Another was using the Multiple Collinearity Scan toolkit (MCScanX) to analyze the gene duplication events, with the default parameters [28].

Ks (synonymous substitution rate) and Ka (nonsynonymous substitution rate) values of tandem duplicated genes were calculated by the method of Nei and Gojobori as implemented in KaKs_calculator [29] based on the coding sequence alignments. The divergence time was calculated based on the formula $\mathrm{T}=\mathrm{Ks} / 2 \mathrm{r}$, with $\mathrm{Ks}$ being the synonymous substitutions per site and $\mathrm{r}$ being the rate of divergence for nuclear genes from plants. $\mathrm{r}$ was taken to be $1.5 \times 10^{-8}$ synonymous substitutions per site per year for dicotyledonous plants [30].

\subsection{Analysis of Cis-Acting Elements}

The upstream $1500 \mathrm{bp}$ of each CsLecRLK was obtained from the genome annotation files of Cucurbit Genomics Databases (http://cucurbitgenomics.org/) via a series of in-house Perl scripts and the cis-acting elements contained in these sequences were then scanned using the Plantcare Databases (http://bioinformatics.psb.ugent.be/webtools/plantcare/html/). In the process of analysis, we filtered out the cis-acting elements that are ubiquitous in most genes, for example CAAT-box, TATA-box, TATC-box and so on, only showing those that may be typical and functional cis-acting elements presented in the CsLecRLK gene family. The structures and annotations of the promoters were then generated using GSDS v.2.0 (http://gsds.cbi.pku.edu.cn/) [28].

\subsection{Expression Pattern Analysis}

To analyze the expression profiles of cucumber CsLecRLK genes in different organs, we retrieved public RNA-seq data (accession number: SRP071224) from the National Center for Biotechnology Information Short Read Archive database. Analysis of RNA-seq data from 10 sampled cucumber tissues was performed based on the regular protocol by Wei et al. [31]. These cucumber tissues included root (4 week old seedlings), hypocotyl (4 week old seedlings), cotyledon (4 week old seedlings), true leaf (4 week old seedlings), stem (12 week old cucumber plants), tendril (12 week old cucumber plants), female flower (12 week old cucumber plants), male flower (12 week old cucumber plants), ovary (unfertilized, 12 week old cucumber plants) and peel (unfertilized ovary, 12-week-old cucumber plants). The Heatmap of the gene Log2 (FPKM + 1) values in 10 tissues of CsLecRLKs was drawn by the R program (3.5.2). To show which genes are widely expressed in different tissues, we also selected five typical tissues, including root (4 week old seedlings), hypocotyl (4 week old seedlings), cotyledon (4 week old seedlings), true leaf (4 week old seedlings) and tendril, to draw Venn diagrams using the $\mathrm{R}$ program (3.5.2).

\subsection{Hormones and Cold Treatments}

The typical cucumber line "9930" was used as the experimental material to investigate the expression pattern in response to various phytohormones and cold treatments. Cucumber seeds were soaked in $55^{\circ} \mathrm{C}$ water for $2 \mathrm{~h}$ and germinated on a petri dish in a growth chamber at $28^{\circ} \mathrm{C}$ in the dark for 2 days. The germinated seeds were grown in pots containing a peat/vermiculite mixture $(3: 1)$ in the greenhouse of Shanghai Jiao Tong University, and the controlled environment growth chamber was programed for light $16 \mathrm{~h} / 25^{\circ} \mathrm{C}$ and dark $8 \mathrm{~h} / 20^{\circ} \mathrm{C}$. After germination for 4 weeks, the seedlings were placed into hydroponic boxes with 1/2 Murashige \& Skoog (MS) liquid solution (pH 5.8, without sugar) for 1 week to adapt to the environment (the root was lucifugal), and were then treated with $100 \mathrm{mM}$ indole-3-acetic acid (IAA), $100 \mathrm{mM}$ 1-naphthaleneacetic acid (NAA), $100 \mathrm{mM}$ abscisic acid (ABA) or 
$100 \mathrm{mM}$ gibberellin (GA) for $3 \mathrm{~h}$ under the same growth conditions as described earlier. The 1/2 MS liquid solution without any hormones was used as a control. Another group of seedlings was treated at $4{ }^{\circ} \mathrm{C}$ for $1 \mathrm{~h}$ and $25^{\circ} \mathrm{C}$ was used as a control. Each treatment consisted of three biological replicates.

\subsection{RNA Extraction and qRT-PCR System}

Roots were collected from plants treated with different hormones, and young leaves (the second node from the top) were collected from plants subject to $4{ }^{\circ} \mathrm{C}$. Total RNA was extracted using the RNeasy Plant Mini Kit (Cwbio, Beijing, China). The first-strand cDNA was prepared according to the PrimeScript RT reagent Kit with gDNA Eraser (Cwbio, Beijing, China) protocol. To identify the relative expression level of different LecRLK genes under different treatments, qRT-PCR was conducted using FastStart Essential DNA Green Master (Roche, Mannheim, Germany). CsActin3 (Csa6G484600.1) was used as an internal control. qRT-PCR was performed in a total volume of $20 \mu \mathrm{L}$, containing $2 \mu \mathrm{L}$ of cDNA, $10 \mu \mathrm{L}$ UltraSYBR mixure (Cwbio, Beijing, China), $2 \mu \mathrm{L}$ gene-specific primers $(10 \mu \mathrm{M})$ and $6 \mu \mathrm{L}$ $\mathrm{ddH}_{2} \mathrm{O}$, using the CFX Connection Real-Time System (Bio-Rad, Hercules, CA, USA) with 40 cycles of $5 \mathrm{~s}$ at $95^{\circ} \mathrm{C}$, and $30 \mathrm{~s}$ at $60^{\circ} \mathrm{C}$. Each experiment was repeated three times, and each experiment included three biological repeats.

\subsection{Primer Design and Data Analysis of qRT-PCR}

Geneious software (version 2019.0.3) was used to design primers according to the cDNA sequences (Supplementary Table S1). PCR-amplified product lengths were about $150 \mathrm{bp}$. The data from real-time PCR amplification were first analyzed using the $2^{-\Delta \Delta C T}$ method [32]. We then divided the result of each gene under different treatments by the result of the control group and obtained the fold change. Statistical differences were determined by a $t$-test $(p<0.05)$ using Microsoft Excel 2010. The heatmap of the fold-change values of CsLecRLKs under five treatments was drawn using the R program (3.5.2).

\section{Results}

\subsection{Genome-Wide Identification of LecRLKs in Cucumber}

We identified a total of 46 LecRLK genes, named CsLecRLKs, in the cucumber genome through Pfam and SMART search, their coding sequence (CDS) and protein sequences are listed in Supplementary Tables S2 and S3. The total number of LecRLKs in cucumber is less than that in Arabidopsis (75 LecRLK genes) or rice (173 LecRLK genes) [4]. The 46 CsLecRLKs were classified into 23 G-type and 22 L-type genes, and one C-type gene based on their extracellular lectin domain. The molecular weights (MWs) of the proteins ranged from 62.5 (Csa1G056960) to $94.5 \mathrm{kDa}$ (Csa3G733860), the isoelectric points (Ips) ranged from 4.98 (Csa4G289630) to 9.51 (Csa1G056960) and the range of CDS length was 1803 to $2502 \mathrm{bp}$. With the predicted protein structures, it could be considered that most of the CsLecRLKs were localized on the plasma membrane, only Csa7G045520 was located on the extracellular. More information for CsLecRLKs, including the length of the gene, the length of CDS, the length of the protein sequence, the protein Molecular weight (MW) and isoelectric point (pI) is listed in Supplementary Table S4.

By analyzing the molecular weight of all 46 CsLecRLKs, we found that the weight of G-type CsLecRLKs (83.2 kDa) is generally more than L-type (62.5 kDa) and C-type (74.6 kDa) CsLecRLKs. This may be mainly due to the fact that in addition to the lectin domain, G-type CsLecRLKs often contain EGF and PAN domains (Figure 1). Signal peptides and transmembrane domains (TMs) are critical for protein localization. The software prediction indicated that not all CsLecRLKs had signal peptides and unique TM domains. The loss of a signal peptide or TM domain directly affected the localization of proteins in cells (Supplementary Table S4). The plasma membrane localization of most of the CsLecRLKs indicated that they are signal receptors that can sense extracellular signals and then transmit the signals to the interior of the cells. 


\subsection{Phylogenetic Analysis of CsLecRLKs}

We constructed a rooted phylogenetic tree using the MEGAX v.10.1.8 (Figure 2). As expected, the phylogenetic tree showed that the CsLecRLK family could be classified into three groups of L-type, G-type and C-type. This result was consistent with the domain-based classification of the CsLecRLK family. The phylogenetic tree indicated that the L-type and C-type groups had a closer relationship. This result was different from previous reports on Arabidopsis and rice, which revealed a closer genetic relationship between G-type and L-type groups [4]. As shown in Figure 2, the phylogram of L-type CsLecRLKs could be divided into four sub-groups respectively, by high bootstrap values. However, G-type CsLecRLKs could be divided into at least eight subgroups by bootstrap values. The main reason for this is that there was a big difference in the N-terminals of G-type CsLecRLKs. Some G-type CsLecRLKs contained an S-domain, EGF domain and PAN domain, while others contained only a bulb lectin domain. At the same time, the three LecRLKs from Arabidopsis were not isolated, but were incorporated into three different types of CsLecRLK, indicating that different types of LecRLK have high specificity among each other.

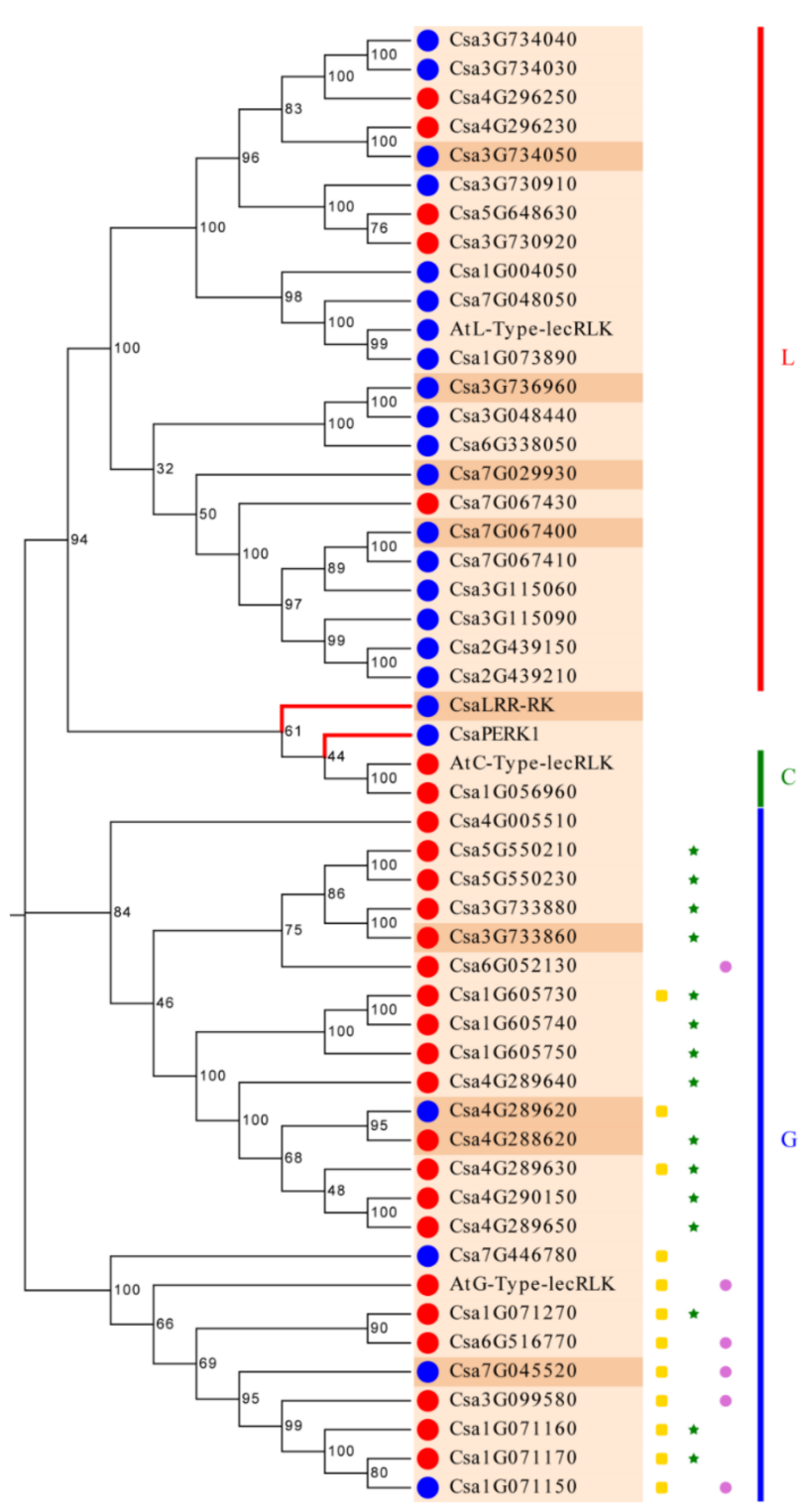

Figure 2. Rooted Phylogenetic tree construction and structure analysis of CsLecRLKs. The L, G and C respectively represent the L-type, G-type and C-type LecRLK subfamilies. The blue circles in front of CsLecRLKs represents proteins with a signal peptide, the red circles in front of CsLecRLKs represented 
protein without a signal peptide. The green stars behind CsLecRLK represents proteins containing Epidermal Growth Factor (EGF) domains, the yellow squares behind CsLecRLKs represents proteins containing Plasminogen Apple Nematode (PAN) domains. The purple circles behind CsLecRLK represents proteins containing $S$ domains. The sandy-brown leaves represents proteins only containing one transmembrane domain, the peach-puff leaves represents proteins containing more than one transmembrane domain.

\subsection{Exon-Intron Structural Analysis of CsLecRLKs}

The genomic sequence and corresponding cDNA sequence of the CsLecRLKs were submitted together to the Gene Structure Display Server (GSDS) together for analyzing their gene structure (Figure 3). The genome sequence lengths of CsLecRLKs ranged from 1803 to $6481 \mathrm{bp}$, and the lengths of CDS ranged from 1674 to $2502 \mathrm{bp}$. The number of exons of these genes varied from one to nine. Similar to the structure of the LecRLK family in other plant genomes $[4,15,16]$, cucumber CsLecRLKs generally lack introns. Eighty percent of the studied CsLecRLKs had less than three exons. Except for Cs4G296230, which contained three exons, all L-type CsLecRLKs contained only one or two exons, and the C-type CsLecRLK (Csa1G056960) contained four exons. The G-type CsLecRLKs contained one to nine exons. In this group, Csa7G446780 contains nine exons, the most exons of all CsLecRLKs analyzed.

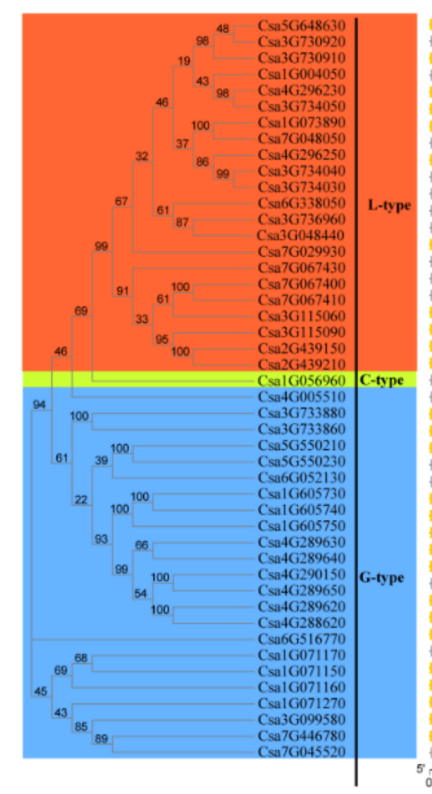

$\mathbf{A}$
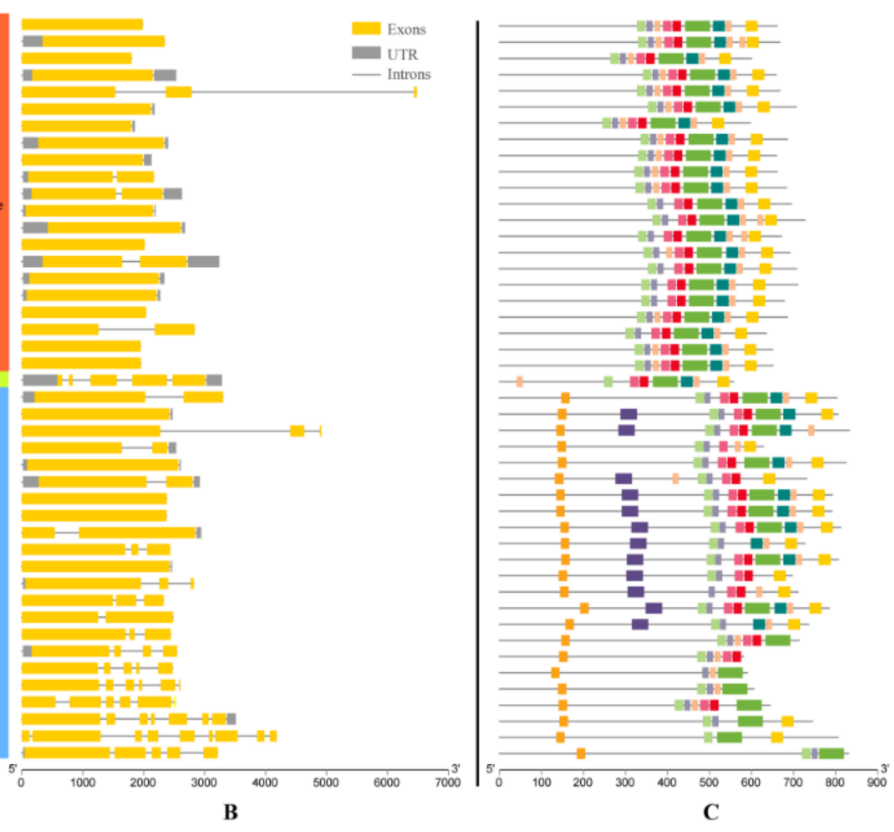

Figure 3. An analytical view of the CsLecRLK genes. (A) Protein maximum likelihood tree. The tree was constructed using a maximum-likelihood method, and bootstrap values were calculated with 1000 replications using MEGAX; (B) Gene structure: The lines represents the introns. The gray squares represents the 3' UTR and the 5' UTR. The yellow square represents the exon; (C) Protein structure. The search for 10 common motifs shared among the CsLecRLK proteins. Different colors represents different motifs.

\subsection{Protein Domain and Motif Analysis of CsLecRLKs}

Through the SMART program prediction, we investigated conserved domains that are present in CsLecRLKs. C-type and L-type CsLecRLKs both only contained three base category domains, Lectin domain, transmembrane and kinase domain. However, some G-type CsLecRLKs also contained two other categories of domains, PAN and EGF domains. Among G-type CsLecRLKs, 10 proteins contained PAN and EGF domains at the same time, 5 proteins only contained PAN domain, 8 proteins only contained the EGF domains, and only one contained neither the PAN domain nor the EGF domain. 
Our result indicates that signal peptides are not necessary in CsLecRLKs. There were 25 CsLecRLKs identified without signal peptides and eight CsLecRLKs with more than two transmembrane domains.

Ten conserved motifs were identified in CsLecRLKs using the MEME program (Figure 3). These motifs were labelled Motif 1 to Motif 10 from the $\mathrm{N}$ - to the C-terminus. The details of the conserved motifs are shown in Supplementary Figure S1. The lengths of these motifs ranged from 15 to 60 residues. Generally, the CsLecRLKs contained 4 to 10 motifs. None of the motifs appeared in all gene family members. Except for Motif 8 and Motif 9, which were only present in the G-type CsLecRLKs, other motifs were present in the three types of CsLecRLKs. With the CDD program (https://www.ncbi.nlm.nih.gov/cdd/), we found that six of these motifs represented different kinase domains (Supplementary Figure S1), indicating that there may be multiple catalytic phosphorylation sites or targets in each of the CsLecRLKs.

\subsection{Chromosomal Location and Gene Duplication of CsLecRLKs}

We extracted the location data of CsLecRLKs and the length data of each chromosome from the cucumber genome annotation files with a series of Perl scripts, and constructed a gene location map using MapChart software. As shown in Figure 4, the CsLecRLKs were unevenly distributed across 7 cucumber chromosomes, and genes from the same subfamily on the same chromosome had a tendency to cluster. The number of CsLecRLKs on each chromosome varied from two to seven, chromosome 3 contains the largest number of 12 CsLecRLKs and chromosome 2 had only two CsLecRLKs.



Figure 4. Chromosomal distribution and gene duplications of CsLecRLK genes. The number on the chromosome (left) represents the position of CsLecRLK genes, and the information on the chromosome (right) represents the gene ID of the CsLecRLKs. The tandem duplicated genes are represented by a yellow background, and the gene clusters are boxed together by black lines. The red represents L-type CsLecRLKs, the green represents C-type CsLecRLKs and the blue represents G-type CsLecRLKs.

During biological evolution, the generation of a gene family may be caused by tandem duplication and segmental duplication $[27,33]$. In order to explore whether the CsLecRLK gene family also had an expansion caused by the two kinds of duplication, we analyzed the duplication events of CsLecRLK genes. The result indicated that although many CsLecRLK genes were clustered on the chromosomes, only Csa1G071170 and Csa1G071160 were a pair of tandem duplicated genes, their divergence was about 38.61 million years ago (MYA). The other two pairs of duplication events, Csa1G073890 and Csa7G048050, and Csa3G734030 and Csa4G296230, may be have been caused by duplication or ectopia of chromosome fragments during the evolution. These duplicated genes were not in the same chromosome. Their divergence times were 30.96 and 32.35 MYA, respectively. Based on the above results, it could be inferred that duplication events contributed to the expansion of the CsLecRLK gene family. 


\subsection{Cis-Acting Elements Analysis on Promoters of CsLecRLKs}

Different genes have their own specific cis-acting elements on their promoters. Transcription factors can bind to the cis-acting elements to regulate the gene expression. Different cis-acting elements may respond to different biotic or abiotic stress signals which could induce or inhibit the genes' expression. Therefore, the cis-acting elements analysis of CsLecRLK promoters will help us to further understand the function of these genes. We used the Plantcare website (http: //bioinformatics.psb.ugent.be/webtools/plantcare/html/) to analyze the promoters of the $1500 \mathrm{bp}$ upstream sequence from the translation initiation site of CsLecRLKs, and found that there were 54 typical and functional cis-acting elements (Supplementary Figure S2), which could be divided into four types: light response, stress resistance, plant hormone and others. Among them, 24 cis-acting elements were related to light response, 11 were related to hormones including salicylic acid (SA), jasmonic acid (JA), ethylene (ET), gibberellin (GA) and auxin, and 9 were abiotic stress elements. These results suggested that the CsLecRLK gene family may be mainly involved in the biological pathway of stress resistance in cucumber. There were six developmentally related cis-acting elements, five of which were related to seed development, suggesting that this gene family may play a role in seed development. More details are shown in Supplementary Table S5.

\subsection{Expression Pattern Analysis of CsLecRLK Genes}

As a first attempt to provide insights into their potential functions, we used RNA-seq data from 10 tissues of cucumber to investigate the expression of each CsLecRLK gene (Supplementary Table S6). Most CsLecRLKs were expressed at a low level, and some (Csa6G338050, Csa1G071160 and Csa3G115090) were barely expressed in any tissue.

RNA-seq analysis showed that the expression of CsLecRLKs was ubiquitous. Each tissue contained at least 25 constitutive expression pattern (FPKM $\geq 1$ ) genes (Figure $5 \mathrm{~A}$ ), except for male flower and unfertilized ovary. The expression pattern of all CsLecRLKs could be divided into 3 groups based on their expression level in each tissue (Figure 5A). From Groups 1 to 3, the range and level of gene expression decreased successively. Group 1 contained seven genes, which had a high expression level in each tissue with an average FPKM of 18.01. There were 12 genes belonging to Group 2, and they had an intermediate expression level in each tissue, with an average FPKM of 6.48. Group 3 included 27 genes expressed at a low level in each tissue with an average FPKM of 1.29. Except for the C-type CsLecRLK (Csa1G056960), which belonged to Group 1, the L-type CsLecRLKs had higher expression levels than the G-type.

Thirty-six CsLecRLKs were expressed in all tissues (FPKM $>0$ ), and 17 genes were constitutively expressed $(F P K M \geq 1)$. Then, we focused on those genes with relatively high expression (FPKM $\geq 2)$ and selected five tissues of cucumbers for cluster analysis (Figure 5B), including root, hypocotyl, cotyledon, true leaf and tendril. We found a total of 16 genes that were expressed in all these tissues. Especially, two genes were expressed only in the roots (Csa2G439210 and Csa3G115060), one gene (Csa3G099580) was expressed in the tendril, and two genes were only expressed in the cotyledon (Csa7G446780 and Csa7G067410). 

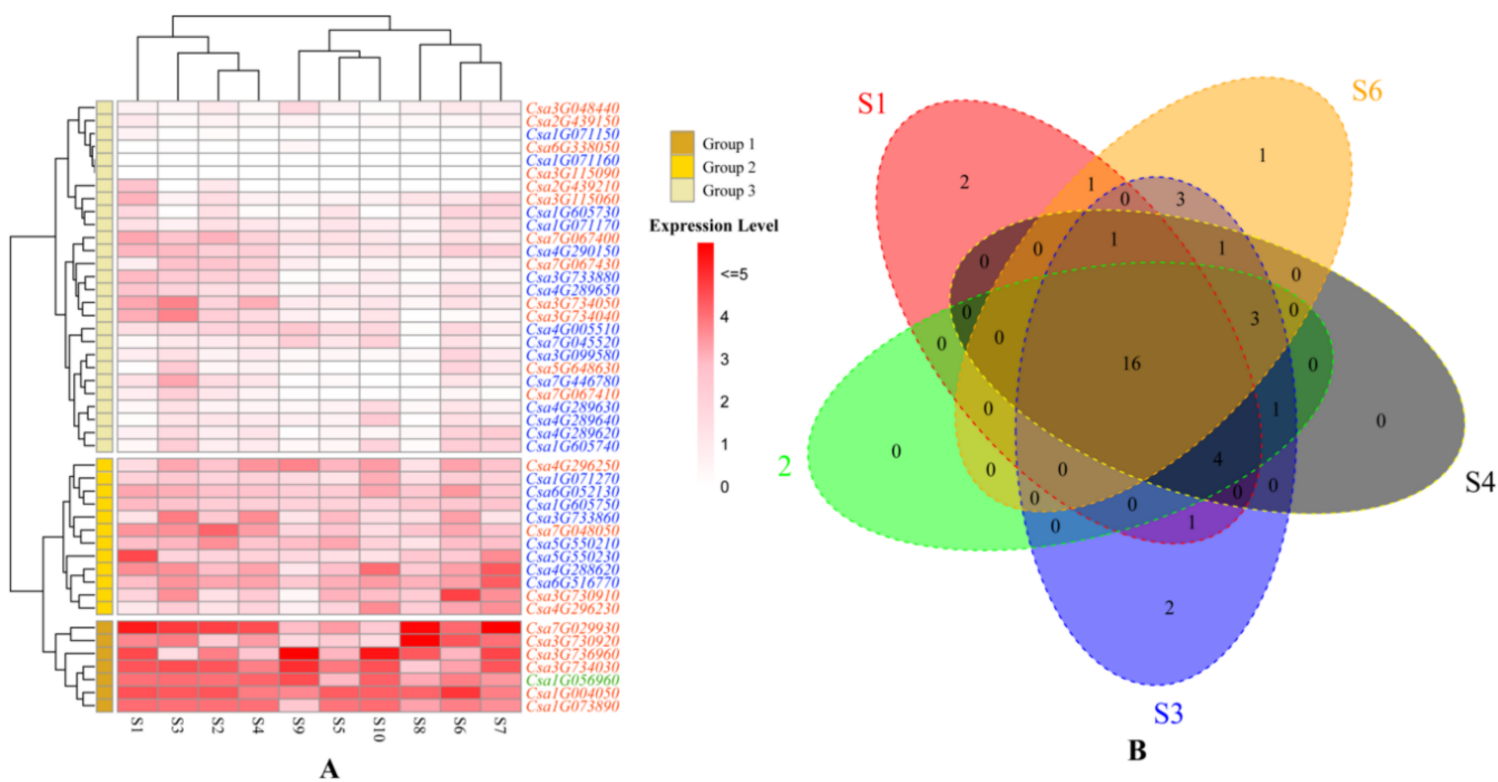

Figure 5. Expression profile of CsLecRLK gene family in different tissues. (A) Heatmap depicting the expression profile of the CsLecRLK family in different developmental tissues. The red represents L-type CsLecRLKs, the green represents C-type CsLecRLKs and the blue represents G-type CsLecRLKs. Genes highly expressed in tissues are colored red, and genes not expressed in tissues are colored blue; (B) Venn diagram depicting the distribution of shared expression of CsLecRLKs in five typical tissues. The following abbreviations are used: S1: roots of 4 week old seedlings; S2: hypocotyl of 4 week old seedlings; S3: cotyledon of 4 week old seedlings; S4: true leaf of 4 week old; S5: Stem; S6, Tendril; S7: female flower; S8: male flower; S9: unfertilized ovary; S10: peel of unfertilized ovary.

\subsection{Expression Analysis of CsLecRLK Genes in Response to Different Treatments}

Gene expression is not only spatiotemporally specific but also can be induced or repressed by hormones and stress. Because most of LecRLKs are receptor proteins on the cell membrane, they can usually sense stimuli at the first time and send signals to intracellular receptors. To uncover all the divergence information for CsLecRLKs in different environments over a short period of time, the expression patterns under different hormone treatments, including IAA, GA, ABA, NAA and cold stress treatments, were analyzed by qRT-PCR. The result showed that most CsLecRLKs (31/46) responded to at least one treatment (Fold-change $>1$ compare to the control group; Significance $p \leq 0.05$ ). Overall, there were 20 upregulated events and 38 downregulated events in total (Significance $p \leq 0.05$ ). In order to show the experimental results more conveniently and intuitively, the fold-change under different treatments is displayed in the heatmap (Figure 6) based on the data of the qRT-PCR. Firstly, some CsLecRLKs (7/46) could be induced or repressed by multiple treatments (treatment number $>3$ ). For instance, Csa1G071170 could be induced by GA, IAA, NAA and ABA treatments, and Csa4G005510 was repressed by all treatments except ABA. Secondly, the expression of different CsLecRLKs could be induced by different treatments. The cold stress induced or repressed some CsLecRLKs genes' expression. The expression level of four genes changed, and they were downregulated. In contrast, NAA induced or repressed the most CsLecRLK genes' expression. There were 20 genes that responded to NAA treatment, 6 genes were upregulated, and 14 genes were downregulated. A total of 16 CsLecRLKs changed their expression level under ABA treatment: 8 genes were upregulated, and 8 genes were downregulated. IAA and GA caused expression level change in nine and eight genes, respectively. The IAA treatment caused one gene's expression to be upregulated, and those of eight genes to be downregulated. The GA treatment caused five genes' expressions to be upregulated and those of three to be downregulated. Thirdly, 14 CsLecRLKs had different expression patterns under various treatments; for example, Csa3G734030 could be induced by NAA, and repressed by ABA, while Csa1G071150 was upregulated under ABA treatment, and downregulated under NAA 
and IAA treatments. The results indicated that the CsLecRLKs have their own response characteristics to hormones and stresses and may play an important role in sensing external stimulus signals. For example, although Csa1G071160 and Csa3G115090 were not expressed in the root, our experiment showed that they can be induced by NAA and ABA, respectively. There were 15 genes that did not have significant expression change under different treatments, they were: Csa1G056960, Csa7G029930, Csa5G550210, Csa4G296250, Csa7G048050, Csa1G073890, Csa4G289620, Csa3G115060, Csa3G099580, Csa1G071270, Csa6G516770, Csa2G439150, Csa1G605730, Csa6G338050 and Csa5G648630.



Figure 6. Expression analysis of CsLecRLK genes in response to different treatments. The heatmap represents the fold-change of expression level under five treatments: gibberellin (GA), abscisic acid (ABA), 1-Naphthaleneacetic acid (NAA), indole-3-acetic acid (IAA) and cold stress.

\section{Discussion}

The number of LecRLK gene family members varies in different plants. In cucumber, 46 LecRLK genes were identified and classified into three groups according to the analysis of phylogenetic relationship. The result was consistent with the classification based on the N-terminal domain differences of the three subfamilies. Compared with other plants, there were fewer members of the LecRLK gene family identified in cucumber. This phenomenon may be due to the following two reasons. Firstly, the genome sizes are diverse among various plants and may affect the number of gene family members. For example, soybean and wheat have more than 1 and 14 GB genomes and contain 52,051 and 39,238 protein-coding genes, respectively [34,35]. Correspondingly, soybean and wheat contain 189 and 263 LecRLK genes, respectively [14,16]. Secondly, compared with other plants, there were fewer 
duplication events in the CsLecRLK gene family in cucumber. Previous studies revealed that Arabidopsis experienced two whole-genome duplications (WGDs), while Populus experienced only one [36,37]. Due to cucumber lacking a recent WGD [38], there have been fewer opportunities for gene families to expand in the form of duplications during evolution in cucumber. In this study, only three pairs of duplicated genes were identified in cucumber. Although the genome size of Arabidopsis ( $125 \mathrm{Mb})$ is smaller than that of in cucumber (367 Mb) [38], Arabidopsis (75 LecRLK genes) has more LecRLKs than cucumber (46 LecRLK genes). The underlying reason may be the WGD that occurred in Arabidopsis. A total of 10 motifs were identified in 46 CsLecRLKs, 6 of them were located at the C-terminal of CsLecRLKs and were related to the catalytic activity of kinases (Figure 3 and Supplementary Figure S1). The result suggested that although the CsLecRLK gene family was divided into three subfamilies according to the $\mathrm{N}$-terminal lectin domain, there may be significant differences in target and kinase activity between members in the same subfamily. At the same time, the C-terminus of each CsLecRLK contains at least three motifs related to the catalytic function of kinase. This also implies that each CsLecRLK has more than one site for catalysis or protein interaction. These results indicated that despite the smaller number of CsLecRLK gene families, they may participate in multiple biological pathways, making up for the shortfall to some extent.

Another interesting finding in this study was that most CsLecRLK genes contained relatively few introns. The gene structure analysis showed that the average intron number per CsLecRLKs was 1.5, which was significantly less than the average intron number (4.39) of cucumber genes [38]. This phenomenon was similar to that observed in previous studies in other plants. For example, most members of the LecRLK gene family in soybean only have one intron or even none at all [16]. A previous study on Arabidopsis and rice also indicated that few genes have introns in the LecRLK gene family. For example, only five and eight genes have introns in Arabidopsis (75 LecRLK genes) and rice (173 LecRLK genes), respectively [4]. The lack of introns may be due to these genes acting as the signal receptors in the plant. The compact gene structure is thought to improve the efficiency of gene expression by reducing variable splicing and saving energy, especially those genes needed to respond to various environmental stimuli [39]. There were six CsLecRLKs that had more introns than the average intron number of cucumber genes, and four of these did not respond to any hormone or cold stimuli in our study. In the cold stress experiment, although the treatment time was only one hour, it still caused significant changes in the expression levels of four genes. Among these genes, Csa4G289630, with the smallest change in expression level, contains two introns, while the other three genes only contained one intron. This evidence further suggests that fewer introns may help LecRLKs respond to stimuli quickly.

RNA-seq analysis showed that the expression of CsLecRLKs is ubiquitous and their expression level in cotyledons, roots, tendrils and hypocotyls were relatively higher than that in other tissues (Figure 6). Cluster analysis showed that the expression of some CsLecRLK genes was tissue-specific. Csa2G439210 was only expressed in roots and cotyledons and its expression level in roots was 5 times higher than that in cotyledons. LECRK-IX.1, the homologous gene of Csa2G439210 in Arabidopsis, was also specifically expressed in roots and guard cells [40]. Csa5G648630 was expressed in tendrils and cotyledons, but hardly expressed in other tissues. Its homologous gene LECRK-VI.1 is mainly expressed in cotyledons and guard cells in Arabidopsis [41]. Csa3G099580 is mainly expressed in cotyledons of cucumber, its homologous gene ARK2 is specifically expressed in cotyledons and vascular bundles in Arabidopsis. In addition to responding to pathogen infection, ARK2 can regulate the development of lateral roots by transferring auxin in a phosphate-starved environment [42]. In this study, the expression level of Csa3G099580 decreased 1.57-fold after IAA treatment. These results indicated that LecRLK genes may have a conserved spatiotemporal expression pattern across different species.

The LecRLK family is a special family in the plant genome; to date, this family has not been found in fungi or humans [43]. As the signal station of plants, a unique characteristic of the LecRLK family may be closely related to its function of sensing the external environment. Previous studies indicated that some LecRLKs are involved in sensing invasion of microorganisms. SD1-29, a G-type LecRLK in 
Arabidopsis, can identify lipopolysaccharides, which are a secretions from Gram-negative Pseudomonas and Xanthomonas bacteria [44]. Some LecRLKs will change their expression pattern with the changes of hormone and nutritional conditions. For example, SIT1, an L-type LecRLK in rice, can mediate salt sensitivity. With increasing $\mathrm{NaCl}$ concentration, SIT1 is activated rapidly, which reduces the survival of rice [45]. In addition, some LecRLKs could affect the development and growth of plants; for instance, two L-type LecRLKs, LecRK-IX.1 and LecRK-IX.2, induce cell death, thereby increasing plant survival when infected with Phytophthora [40]. Analysis of hormone and cold treatments showed that most CsLecRLKs (31/46) responded to at least one treatment (Fold-change $>1$ ). We also found a large number of cis-acting elements closely related to stress and stimulus response that existed in promoter regions of CsLecRLK genes (Supplementary Table S5). These results indicate that the CsLecRLK gene family may play a key role in responding to environmental stimuli. For example, there were three cis-acting elements related to abscisic acid (ABA) response in the promoter of Csa4G296230 (G-type CsLecRLK). Its expression level was upregulated 88-fold in a short period after ABA treatment. The promoter of Csa5G550210 (L-type CsLecRLK) also contains a regulatory element related to abscisic acid response. After ABA treatment, its expression was downregulated 17-fold. An auxin-related regulatory element exists in the promoter of Csa4G289650, and expression level of Csa4G289650 was downregulated 5-fold under IAA treatment. Certainly, although the expression of some CsLecRLKs was not detected in tested tissues or not induced by hormones and stress treatments in this study, it may be induced by other hormones or stress, such as salicylic acid, ethylene, salt stress, insect stress and so on. These induction methods and gene function analyses of response to hormones or stress will be the research direction in the future.

\section{Conclusions}

In this study, a total of 46 CsLecRLKs were identified using bioinformatics methods, and most of them lacked introns. Forty-six CsLecRLKs were clustered phylogenetically into three distinct subfamilies, and C-type CsLecRLKs and L-type CsLecRLKs had a close relationship. Through the cis-elements analysis, many regulatory elements associated with phytohormones and stress were found on the CsLecRLKs' promoters. Transcriptome data demonstrated distinct expression patterns of CsLecRLK genes in various tissues. In different tissues, most CsLecRLKs were expressed at a low level, and three CsLecRLKs were barely expressed in any tissue. In addition, the quantitative real-time PCR (qRT-PCR) analysis showed that each member of the CsLecRLK gene family had its own unique expression pattern under hormone and stress treatment. The information obtained in the current study could help to provide a clear understanding of the features of the LecRLK gene family in cucumber.

Supplementary Materials: The following are available online at http://www.mdpi.com/2073-4425/11/9/1032/s1, Figure S1: Conserved motif structure analyses of CsLecRLKs, Figure S2: Promoter Analysis of CsLecRLKs, Table S1: Primers for qRT-PCR in this study, Table S2: CDS sequences of CsLecRLKs, Table S3: Protein sequences of CsLecRLKs, Table S4. Basic character of CsLecRLK family, Table S5: Promoter elements analysis of 46 CsLecRLK genes, Table S6: RNA-Seq data of 46 CsLecRLK genes in different tissues.

Author Contributions: Conceptualization, J.-S.P.; RNA extraction and qRT-PCR, L.-R.X. and Y.Y.; Resources, J.-X.S., H.-L.H. and R.C.; Software, Y.C. and C.-L.G.; Data analyses, D.L.; Validation, J.-S.P.; Writing-original draft, D.L.; Writing - review and editing, D.L., G.W. and J.-S.P. All authors have read and agreed to the published version of the manuscript.

Funding: This study was supported by the National Natural Science Foundation of China (No. 31672148) and the Foundation of Shanghai Municipal Commission of Science and Technology (Grant No.18391900300).

Acknowledgments: We thank Ling Dai (School of Electronic Information and Electrical Engineering, Shanghai Jiao Tong University, China) for helping with bioinformatics analysis, and Wencan Zhang for revising the manuscript (School of Naval Architecture, Ocean and Civil Engineering, Shanghai Jiao Tong University, China).

Conflicts of Interest: The authors declare no conflict of interest and the sponsors had no role in the design, execution, interpretation, or writing of the study. 


\section{References}

1. Guo, J.; Duan, H.; Xuan, L.; Wang, Z.; Yang, Y. Identification and functional analysis of LecRLK genes in Taxodium 'Zhongshanshan'. PeerJ 2019, 7, e7498. [CrossRef] [PubMed]

2. Shiu, S.H.; Bleecker, A.B. Receptor-like kinases from Arabidopsis form a monophyletic gene family related to animal receptor kinases. Proc. Natl. Acad. Sci. USA 2001, 98, 10763-10768. [CrossRef]

3. Klaas, B.; Francine, G. Arabidopsis L-type lectin receptor kinases: Phylogeny, classification, and expression profiles. J. Exp. Bot. 2009, 15, 4383-4396.

4. Vaid, N.; Pandey, P.K.; Tuteja, N. Genome-wide analysis of lectin receptor-like kinase family from Arabidopsis and rice. Plant Mol. Biol. 2012, 80, 365-388. [CrossRef] [PubMed]

5. Vaid, N.; Macovei, A.; Tuteja, N. Knights in action: Lectin receptor-like kinases in plant development and stress responses. Mol. Plant 2013, 6, 1405-1418. [CrossRef]

6. Tanksley, S.D.; Loaiza-Figueroa, F. Gametophytic self-incompatibility is controlled by a single major locus on chromosome 1 in Lycopersicon peruvianum. Proc. Natl. Acad. Sci. USA 1985, 82, 5093-5096. [CrossRef]

7. Shiu, S.H. Plant receptor-like kinase gene family: Diversity, function, and signaling. Sci. STKE 2001, 2001, e22. [CrossRef]

8. Naithani, S.; Chookajorn, T.; Ripoll, D.R.; Nasrallah, J.B. Structural modules for receptor dimerization in the S-locus receptor kinase extracellular domain. Proc. Natl. Acad. Sci. USA 2007, 104, 12211-12216. [CrossRef]

9. Hervé, C.; Serres, J.; Dabos, P.; Canut, H.; Barre, A.; Rougé, P.; Lescure, B. Characterization of the Arabidopsis lecRK-a genes: Members of a superfamily encoding putative receptors with an extracellular domain homologous to legume lectins. Plant Mol. Biol. 1999, 39, 671-682. [CrossRef]

10. Cambi, A.; Koopman, M.; Figdor, C.G. How C-type lectins detect pathogens. Cell. Micro. Biol. 2005, 7, 481-488. [CrossRef]

11. Deng, K.; Wang, Q.; Zeng, J.; Guo, X.; Zhao, X.; Tang, D.; Liu, X. A Lectin receptor kinase positively regulates ABA response during seed germination and is involved in salt and osmotic stress response. J. Plant Biol. 2009, 52, 493. [CrossRef]

12. Wan, J.; Patel, A.; Mathieu, M.; Kim, S.Y.; Xu, D.; Stacey, G. A lectin receptor-like kinase is required for pollen development in Arabidopsis. Plant Mol. Biol. 2008, 67, 469-482. [CrossRef] [PubMed]

13. Cheng, X.; Wu, Y.; Guo, J.; Du, B.; Chen, R.; Zhu, L.; He, G. A rice lectin receptor-like kinase that is involved in innate immune responses also contributes to seed germination. Plant J. 2013, 76, 687-698. [CrossRef] [PubMed]

14. Shumayla; Shailesh, S.; Pandey, A.K.; Kashmir, S.; Kumar, U.S.; Manoj, P. Molecular characterization and global expression analysis of lectin receptor kinases in bread wheat (triticum aestivum). PLoS ONE 2016, 11, e153925. [CrossRef] [PubMed]

15. Yang, Y.; Labbé, J.; Muchero, W.; Yang, X.; Jawdy, S.S.; Kennedy, M.; Johnson, J.; Sreedasyam, A.; Schmutz, J.; Tuskan, G.A. Genome-wide analysis of lectin receptor-like kinases in Populus. BMC Genom. 2016, 17, 1-16. [CrossRef]

16. Liu, P.L.; Huang, Y.; Shi, P.H.; Yu, M.; Xie, J.B.; Xie, L.L. Duplication and diversification of lectin receptor-like kinases (LecRLK) genes in soybean. Sci. Rep. 2018, 8, 5861. [CrossRef]

17. Wu, T.; Wang, R.; Xu, X.; He, X.; Sun, B.; Zhong, Y.; Liang, Z.; Luo, S.; Lin, Y. Cucumis sativus L-type lectin receptor kinase (CsLecRK) gene family response to Phytophthora melonis, Phytophthora capsici and water immersion in disease resistant and susceptible cucumber cultivars. Gene 2014, 549, 214-222. [CrossRef]

18. Johnson, L.S.; Eddy, S.R.; Portugaly, E. Hidden Markov model speed heuristic and iterative HMM search procedure. BMC Bioinform. 2010, 11, 431. [CrossRef]

19. Finn, R.D.; Coggill, P.; Eberhardt, R.Y.; Eddy, S.R.; Mistry, J.; Mitchell, A.L.; Potter, S.C.; Punta, M.; Qureshi, M.; Sangrador-Vegas, A.; et al. The Pfam protein families database: Towards a more sustainable future. Nucleic Acids Res. 2016, 44, D279-D285. [CrossRef]

20. Ivica, L.; Peer, B. 20 years of the SMART protein domain annotation resource. Nucleic Acids Res. 2017, D1. [CrossRef]

21. Rc, E. Muscle: Multiple sequence alignment with high accuracy and high throughput. Nucleic Acids Res. 2004, 32. [CrossRef]

22. Timothy, L.B.; Nadya, W.; Chris, M.; Wilfred, W.L. Meme: Discovering and analyzing DNA and protein sequence motifs. Nucleic Acids Res. 2006, 34. [CrossRef] 
23. Hu, B.; Jin, J.; Guo, A.Y.; Zhang, H.; Gao, G. GSDS 2.0: An upgraded gene feature visualization server. Bioinformatics 2014, 31, 1296-1297. [CrossRef]

24. Voorrips, R.E. MapChart: Software for the graphical presentation of linkage maps and QTLs. J. Hered. 2002, 93, 77-78. [CrossRef]

25. Gu, Z.; Andre, C.; Chen, F.C.; Peter, B.; Li, W.H. Extent of gene duplication in the genomes of drosophila, nematode, and yeast. Mol. Biol. Evol. 2002, 19, 256-262. [CrossRef]

26. Yang, S.; Zhang, X.; Yue, J.X.; Tian, D.; Chen, J.Q. Recent duplications dominate NBS-encoding gene expansion in two woody species. Mol. Genet. Genom. 2008, 280, 187-198. [CrossRef] [PubMed]

27. Mehan, M.R.; Freimer, N.B.; Ophoff, R.A. A genome-wide survey of segmental duplications that mediate common human genetic variation of chromosomal architecture. Hum. Genom. 2004, 1, 335-344. [CrossRef] [PubMed]

28. Yupeng, W.; Haibao, T.; Jeremy, D.D.; Xu, T.; Jingping, L.; Xiyin, W.; Tae-ho, L.; Huizhe, J.; Barry, M.; Hui, G.; et al. MCScanX: A toolkit for detection and evolutionary analysis of gene synteny and collinearity. Nucleic Acids Res. 2012, 40. [CrossRef]

29. Zhang, Z.; Li, J.; Zhao, X.-Q.; Wang, J.; Wong, G.K.-S.; Yu, J. KaKs_Calculator: Calculating Ka and Ks through model selection and model averaging. Genom. Proteom. Bioinform. 2006, 4, 259-263. [CrossRef]

30. Koch, M.A.; Haubold, B.; Mitchell-Olds, T. Comparative evolutionary analysis of chalcone synthase and alcohol dehydrogenase loci in Arabidopsis, Arabis, and related genera (Brassicaceae). Mol. Biol. Evol. 2000, 17, 1483-1498. [CrossRef]

31. Wei, G.; Tian, P.; Zhang, F.; Qin, H.; Wang, G. Integrative analyses of non-targeted volatile profiling and transcriptome data provide molecular insight into VOC diversity in cucumber plants (cucumis sativus L.). Plant Physiol. 2016, 172, 603-618. [CrossRef] [PubMed]

32. Schmittgen, T.D.; Zakrajsek, B.A.; Mills, A.G.; Gorn, V.; Singer, M.J.; Reed, M.W. Quantitative reverse transcription-polymerase chain reaction to study mRNA decay: Comparison of endpoint and real-time methods. Anal. Biochem. 2000, 285, 194-204. [CrossRef] [PubMed]

33. Kent, W.J.; Baertsch, R.; Hinrichs, A.S.; Miller, W.; James, K.; Robert, B.; Angie, H.; Webb, M.; David, H. Evolution's cauldron: Duplication, deletion, and rearrangement in the mouse and human genomes. Proc. Natl. Acad. Sci. USA 2003, 100, 11484-11489. [CrossRef]

34. Shen, Y.; Liu, J.; Geng, H.; Zhang, J.; Liu, Y.; Zhang, H.; Xing, S.; Du, J.; Ma, S.; Tian, Z. De novo assembly of a Chinese soybean genome. Sci. China Life Sci. 2018, 61, 871-884. [CrossRef] [PubMed]

35. Chaudhary, C. shifting the limits in wheat research and breeding using a fully annotated reference genome. Science 2018, 361. [CrossRef]

36. Bowers, J.E.; Chapman, B.A.; Rong, J.; Paterson, A.H. Unravelling angiosperm genome evolution by phylogenetic analysis of chromosomal duplication events. Nature 2003, 422, 433-438. [CrossRef] [PubMed]

37. Tuskan, G.A.; DiFazio, S.; Jansson, S.; Bohlmann, J.; Grigoriev, I.V.; Hellsten, U.; Putnam, N.H.; Ralph, S.; Rombauts, S.; Salamov, A.; et al. The genome of black cottonwood, Populus trichocarpa (Torr. \& Gray). Science 2006, 313, 1596-1604. [CrossRef]

38. Sanwen, H.; Ruiqiang, L.; Zhonghua, Z.; Li, L.; Xingfang, G.; Wei, F.; William, J.L.; Xiaowu, W.; Bingyan, X.; Peixiang, N.; et al. The genome of the cucumber, Cucumis sativus L. Nat. Genet. 2009, 41, 1275-1281. [CrossRef]

39. Jeffares, D.C.; Penkett, C.J.; Bähler, J. Rapidly regulated genes are intron poor. Trends Genet. 2008, 24, 375-378. [CrossRef]

40. Wang, Y.; Cordewener, J.H.; America, T.; Shan, W.; Bouwmeester, K.; Govers, F. Arabidopsis lectin receptor kinases LecRK-IX.1 and LecRK-IX.2 are functional analogs in regulating phytophthora resistance and plant cell death. Mol. Plant Microbe Interact. 2015, 28. [CrossRef]

41. Ascencio-Ibanez, J.T.; Sozzani, R.; Lee, T.J.; Chu, T.M.; Wolfinger, R.D.; Cella, R.; Hanley-Bowdoin, L. Global analysis of Arabidopsis gene expression uncovers a complex array of changes impacting pathogen response and cell cycle during geminivirus infection. Plant Physiol. 2008, 148, 436-454. [CrossRef]

42. Deb, S.; Sankaranarayanan, S.; Wewala, G.; Widdup, E.; Samuel, M.A. The S-domain receptor kinase arabidopsis receptor Kinase2 and the $\mathrm{U}$ box/armadillo repeat-containing E3 ubiquitin Ligase9 module mediates lateral root development under phosphate starvation in arabidopsis. Plant Physiol. 2014, 165, 1647-1656. [CrossRef] [PubMed] 
43. Paonessa, F.; Criscuolo, S.; Sacchetti, S.; Amoroso, D.; Benfenati, F. Regulation of neural gene transcription by optogenetic inhibition of the RE1-silencing transcription factor. Proc. Natl. Acad. Sci. USA 2015, 113, 2812-2818. [CrossRef] [PubMed]

44. Ranf, S.; Gisch, N.; Schäffer, M.; Illig, T.; Westphal, L.; Knirel, Y.A.; Carballo, P.M.S.; Zähringer, U.; Hückelhoven, R.; Lee, J.; et al. A lectin S-domain receptor kinase mediates lipopolysaccharide sensing in Arabidopsis thaliana. Nat. Immunol. 2015, 16, 426-433. [CrossRef] [PubMed]

45. Li, C.H.; Wang, G.; Zhao, J.L.; Zhang, L.Q.; Ai, L.F.; Han, Y.F.; Sun, D.Y.; Zhang, S.W.; Sun, Y. The receptor-like kinase SIT1 mediates salt sensitivity by activating MAPK3/6 and regulating ethylene homeostasis in rice. Plant Cell 2014, 26, 2538-2553. [CrossRef] [PubMed]

(C) 2020 by the authors. Licensee MDPI, Basel, Switzerland. This article is an open access article distributed under the terms and conditions of the Creative Commons Attribution (CC BY) license (http://creativecommons.org/licenses/by/4.0/). 